Bull. Mater. Sci., Vol. 17, No. 7, December 1994, pp. 1401-1409. (C) Printed in India.

\title{
Selective semiconductor sensors for reducing and oxidizing gases
}

\author{
G S V COLES \\ Department of Electrical and Electronic Engineering, University of Wales, Swansea, \\ SA2 8PP, UK
}

\begin{abstract}
The ever-increasing need for sensors capable of detecting and monitoring toxic and flammable gases is presented and the various techniques available are introduced. Semiconductor devices based on tin dioxide, which potentially have many desirable characteristics, are discussed in detail with particular reference to the work in Swansea which has focused on the problem of selectivity.
\end{abstract}

Keywords. Gas detection; tin dioxide; selective sensors.

\section{Introduction}

There can be no doubt that the need for sensors to monitor a wide variety of measurands will continue to grow. This is illustrated in figure 1 which shows the predicted growth in the European sensor market for the decade from 1990. The world market will undergo similar growth and is expected to be worth in excess of $£ 24$ billion by the year 2000 (Harmer Associates). The market in the specific area of gas sensors is expected to undergo a similar percentage increase, the need occurring in an extremely wide variety of areas including the mining, petrochemical and gas industries; emission monitors; automotive sensors; and medical and domestic applications. This is due in part to increased public awareness, but is mainly a result of associated legislation. These requirements have, in turn, led to a vast amount of world-wide interest in gas sensor research and development. Gas sensor research is one of the truly interdisciplinary fields in modern science calling upon the expertise of, amongst others, chemists, physicists, and electrical and mechanical engineers. There are currently several technologies available for the detection of gaseous species and these are listed in table 1 together with a brief summary of their mode of operation. All of these sensor types possess at least some of the characteristics that are desirable in commercially viable devices, including high sensitivity, fast response, low power consumption, low ambient temperature and humidity dependence, negligible drift, longevity, low cost and selectivity. It should be noted however that few, if any, sensors possess all of these characteristics and that the precise requirements for any sensor will depend on the specific application. For example, if a sensor is required to monitor a toxic gas such as carbon monoxide $(\mathrm{CO})$ then it is usually the threshold limit value [TLV, the time-weighted average concentration to which exposure is considered safe for a specified period (in this case $8 \mathrm{~h}$, i.e. a normal working day)] that is of interest, which in this case is $50 \mathrm{ppm}$. For other gases this can be much lower, for example nickel carbonyl $\left(\mathrm{Ni}(\mathrm{CO})_{4}\right)$ where the $\mathrm{TLV}$ is $0.001 \mathrm{ppm}$. In these cases it is necessary to have a highly sensitive sensor capable of monitoring such low levels. Conversely, if flammable gases are to be detected then it is usually the lower explosive limit (LEL, the concentration in air above which an explosion is possible given a 


\section{European sensor market by parameter}

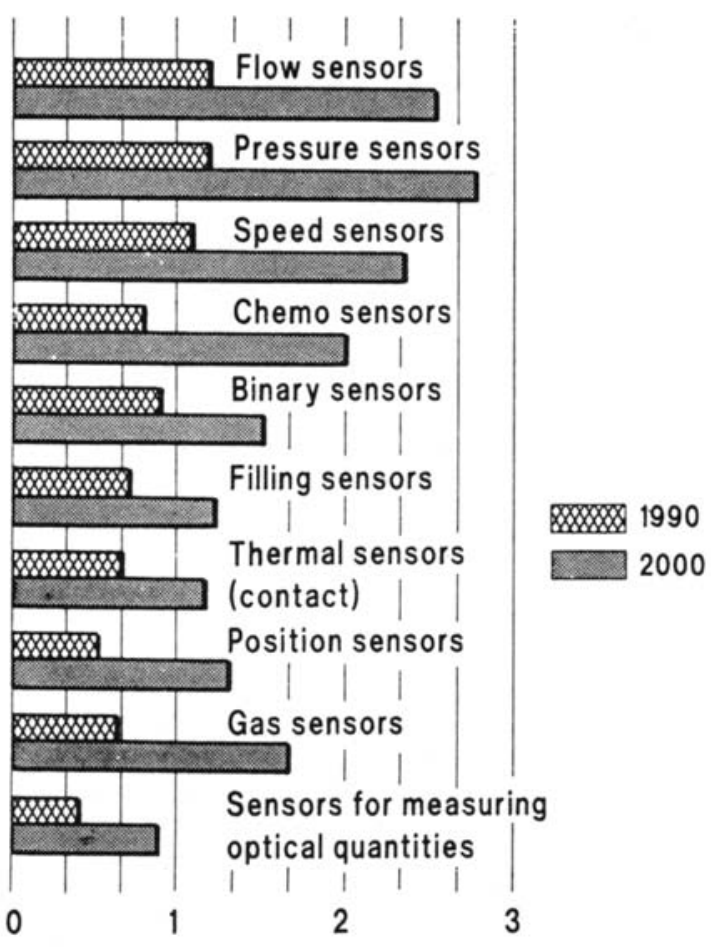

\section{Ebillion}

Figure 1. The expected growth in the European sensor market by parameter for the decade from 1990.

source of ignition) which is of interest and for methane $\left(\mathrm{CH}_{4}\right)$ this is $4 \%$ or $40,000 \mathrm{ppm}$. It is feasible that sensors could be used in these situations where the sensitivity is at least three orders of magnitude less than in the previous cases. Another example is that of power consumption. For an installed instrument or system where mains power is readily available then power consumption is generally a minor consideration. However, the requirement for low-power devices becomes critical if portable, battery operation is envisaged.

The last category of sensors described in the table are the semiconductor devices, which are usually based on tin dioxide $\left(\mathrm{SnO}_{2}\right)$. These devices are amongst the world's best selling type of gas sensor and they have the potential to exhibit many of the previously cited characteristics. However, one of their major disadvantages has been a lack of selectivity which has led to their being ignored by large numbers of sensor users. It is this specific problem that has been studied in Swansea.

\section{Tin dioxide as a gas sensing element}

Tin dioxide occurs naturally as cassiterite which has a rutile-type structure. Its main industrial uses are in the ceramics industry where it is used as an opacifier and 
Table 1. Common types of gas sensor and their mode of operation.

\begin{tabular}{ll}
\hline Gas sorption & where a gaseous species is irreversibly \\
adsorbed onto a compound, often by way \\
of a chemical reaction producing a change \\
in one of the physical properties of the \\
material, such as colour \\
for example, infrared or ultraviolet spectro- \\
photometry in which a specific molecular \\
transition is monitored; this (in addition to \\
the first method) is becoming increasingly \\
linked with fibre-optic technology \\
in which gases react on a catalytic filament \\
via an exothermic process, the resulting \\
temperature increase being monitored via \\
a corresponding resistance change in the \\
Calament \\
where gases react at electrodes to produce \\
electric currents \\
Ion-selective electrodes
\end{tabular}

pigment base, and in the electronics industry where it is used as the transparent electrodes in various applications such as liquid crystal displays. It is a wide-band-gap semiconductor $(\Delta E \sim 3.5 \mathrm{eV})$ in which oxygen vacancies confer n-type properties. When exposed to air at ambient or elevated temperatures oxygen is adsorbed from the air in one or more of the following forms: $\mathrm{O}^{-}, \mathrm{O}^{2-}, \mathrm{O}_{2}^{-}$or $\mathrm{O}_{2}^{2-}$. These oxygen species are available to react with reducing gases, the associated electrons being returned to the conduction band resulting in a resistance decrease. It is this change in resistance that is monitored electrically to give a warning light, alarm or readout. Following reaction, oxygen is again adsorbed from the air and the process continues. This can be visualized as a cyclic reaction, and is shown diagrammatically in figure 2 for the case of carbon monoxide $(\mathrm{CO})$, where it is oxidized to produce carbon dioxide $\left(\mathrm{CO}_{2}\right)$. The problem is that many other reducing gases can also be oxidized at the surface resulting in similar resistance changes. Several mathematical models have also been proposed which describe the operation of these devices (Clifford and Tuma 
1982; Morrison 1987). Oxidizing gases can also affect the resistance of this material by competing with the oxygen adsorption process. In these cases resistance increases are usually observed.

Early work in this area concentrated on achieving a degree of selectivity by temperature control. This is shown in figure 3 for a pure tin dioxide sample (Firth et al 1975). At low temperatures tin dioxide is too resistive to be useful as a sensor element and the reactions are too slow to be of practical use. At high temperatures the response to any gas becomes undetectable due to the low resistivity arising from thermal promotion of electrons to the conduction band. As temperature is increased from ambient a response to $\mathrm{CO}$ is observed above $\sim 200^{\circ} \mathrm{C}$ and this increases, reaching a peak at $\sim 400^{\circ} \mathrm{C}$. No response to methane is observed below temperatures of $300^{\circ} \mathrm{C}$ and the response to this gas peaks at $\sim 500^{\circ} \mathrm{C}$. From figure 3 it can therefore be seen that operating a sensor composed of tin dioxide at a temperature of $\sim 300^{\circ} \mathrm{C}$ gives a sensor which will selectively detect $\mathrm{CO}$ in preference to $\mathrm{CH}_{4}$. It should be noted however that at no temperature is the opposite selectivity observed for this sample.

From this description it can be seen that it is necessary to operate devices of this type at elevated temperatures typically in the range $200-400^{\circ} \mathrm{C}$. This requires the use of a substrate which provides mechanical support for the active material and incorporates both a heater and means of interrogating the material. A typical commercially available substrate is shown in figure 4 . Deposited on one face of the

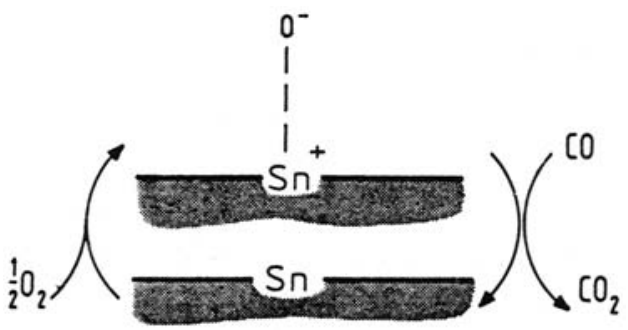

Figure 2. A diagrammatic representation of the reaction occurring on a tin dioxide surface for the case of carbon monoxide.

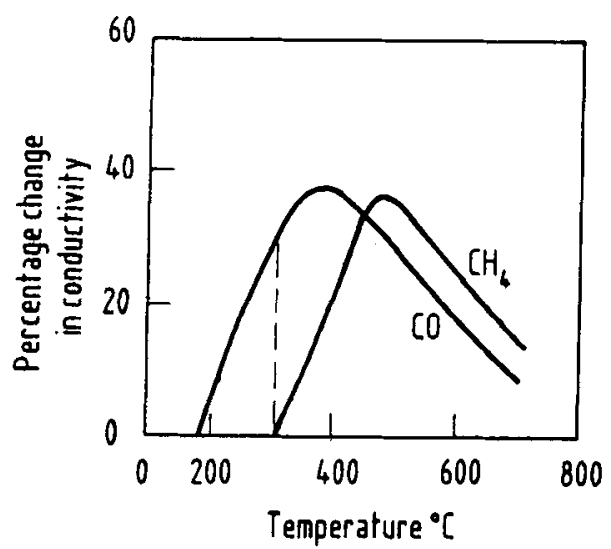

Figure 3. The percentage change in conductivity versus temperature for a pure tin dioxide gas sensor in $100 \mathrm{ppm}$ inclusions of carbon monoxide and methane. 
PLATINUM RESISTANCE HEATER

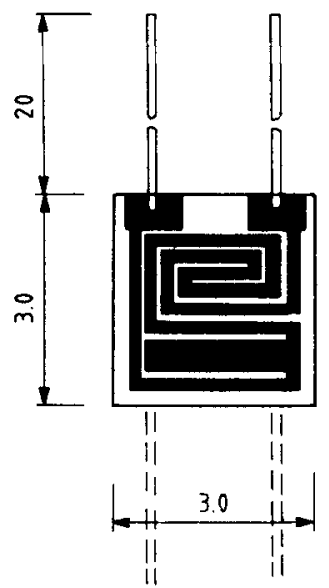

REVERSE SIDE - OPTION A

Interdigitated array

4 pairs of lines and gaps 0.125 wide

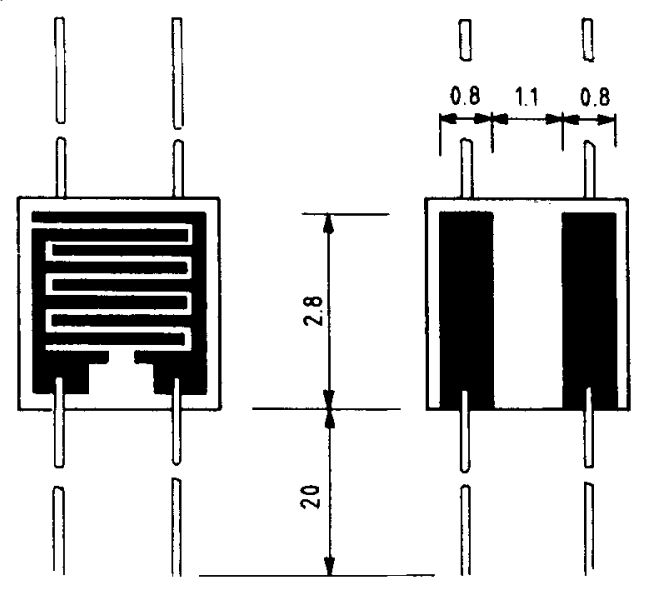

Figure 4. A commercially available sensor substrate (produced by Rosemount Engineering, Bognor Regis, UK) showing the heater track and contact array options.

$3 \mathrm{~mm} \times 3 \mathrm{~mm} \times 0.6 \mathrm{~mm}$ alumina chip is a platinum resistance heater and on the other side a choice of either two parallel platinum pads or an interdigitated array. The latter option is used if active materials with higher resistances are to be measured. These substrates are easily capable of reaching desired operating temperatures and to achieve a temperature of $\sim 300^{\circ} \mathrm{C}$ require about 1 watt. One of the major problems associated with the substrate is price. The active sensor material itself costs fractions of a penny whereas the above substrates retail at $\sim £ 40$. Several groups around the world, including our own, are therefore looking at methods of producing cheaper in-house substrates for gas sensors.

\section{Selective sensors produced at Swansea}

In recent years four selective sensors have been developed at Swansea. The first is sensitive to the presence of carbon monoxide but shows no response to the lower hydrocarbons methane, ethane, propane and butane (Coles et al 1985). This device is produced by sintering an intimate mixture of tin and bismuth oxides. A second sensor exhibits a selectivity opposite to this and is composed of tin dioxide, aluminium silicate and palladium chloride (Coles et al 1985). The third, which is essentially composed of the same materials as the first device, is sensitive to the presence of hydrogen but shows little response to the presence of carbon monoxide or lower hydrocarbons (Coles et al 1991). The fourth device, which is composed of pure tin dioxide but produced via a novel route, detects the environmentally sensitive gas nitrogen dioxide $\left(\mathrm{NO}_{2}\right)$ but is insensitive to any of the previously cited gases (Coles and Williams 1993). The important feature to note with regard to all four sensors is that this selectivity is not produced by temperature control, the sensors exhibiting this behaviour throughout the viable operating temperature range. There will however be an optimum temperature at which the sensors will have maximum sensitivity and 
therefore an optimum power at which the substrates should be heated. It is important to remember that the power required to reach a given temperature is predominantly dependent on substrate size and geometry.

As an example the carbon monoxide-selective sensor will be discussed. Figure 5 shows the variation in conductivity with increasing contaminant gas concentration for a $\mathrm{CO}$-selective device in both $\mathrm{CO}$ and $\mathrm{CH}_{4}$. It can be seen that the sensor responds markedly to even low levels of $\mathrm{CO}$ whilst the response to $\mathrm{CH}_{4}$ remains negligible. In fact the response remains negligible well beyond the boundaries of this figure, up to several thousand ppm which for safety reasons were the limits of these tests. The maximum sensitivity is observed at an operating temperature of $\sim 300^{\circ} \mathrm{C}$. As has been previously stated this selectivity is observed over a wide range of temperature. Figure 6 shows the effect of increasing the amount of bismuth oxide in tin dioxidebased sensors sintered at $800^{\circ} \mathrm{C}$ for $2 \mathrm{~h}$ with $\sim 2^{\circ} \mathrm{C}$ per minute furnace heating and cooling. As expected pure tin dioxide responds to both $\mathrm{CO}$ and $\mathrm{CH}_{4}$, but as the amount of $\mathrm{Bi}_{2} \mathrm{O}_{3}$ is increased the response to $\mathrm{CH}_{4}$ rapidly diminishes, and above $\sim 16 \% \mathrm{w} / \mathrm{w} \mathrm{Bi}{ }_{2} \mathrm{O}_{3}$ there is no response to this gas. Conversely, the response to $\mathrm{CO}$ remains unaltered up to $\sim 18 \% \mathrm{Bi}_{2} \mathrm{O}_{3}$. Above this level a similar reduction in response is observed, in this case reaching zero at bismuth oxide concentrations in excess of $\sim 23 \%$. It can therefore be seen that the ideal composition for this $\mathrm{CO}$ selective device is $17 \%$ bismuth oxide with $83 \%$ tin dioxide where the response to $\mathrm{CO}$ is still at its maximum whilst the response to $\mathrm{CH}_{4}$ is negligible. This is a curious observation as tin dioxide responds to numerous gases but bismuth oxide is known to show little response as a gas sensor. However, this device is sintered at elevated temperatures and it is therefore important to know what happens to the device under these conditions. Figure 7 shows a series of X-ray diffraction scans for a mixture of

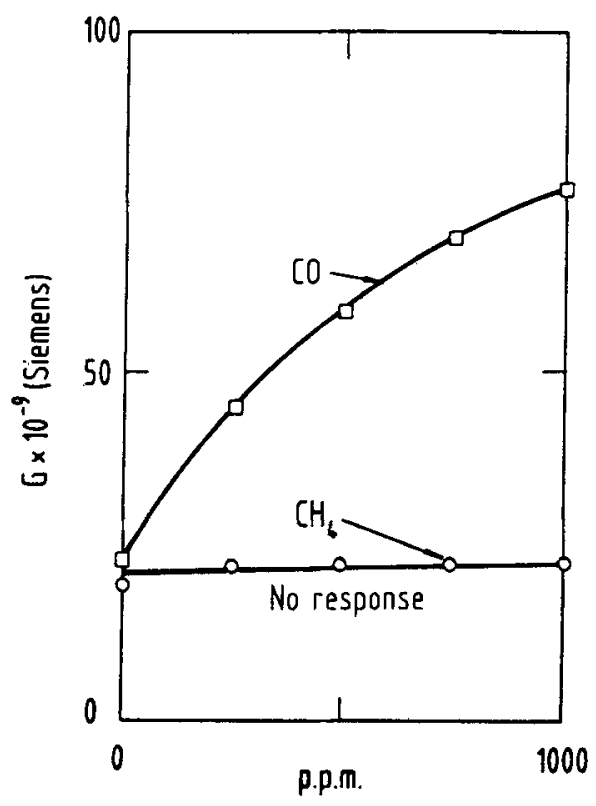

Figure 5. The response of a CO-selective device with increasing gas concentration in atmospheres containing $\mathrm{CO}$ and $\mathrm{CH}_{4}$. 


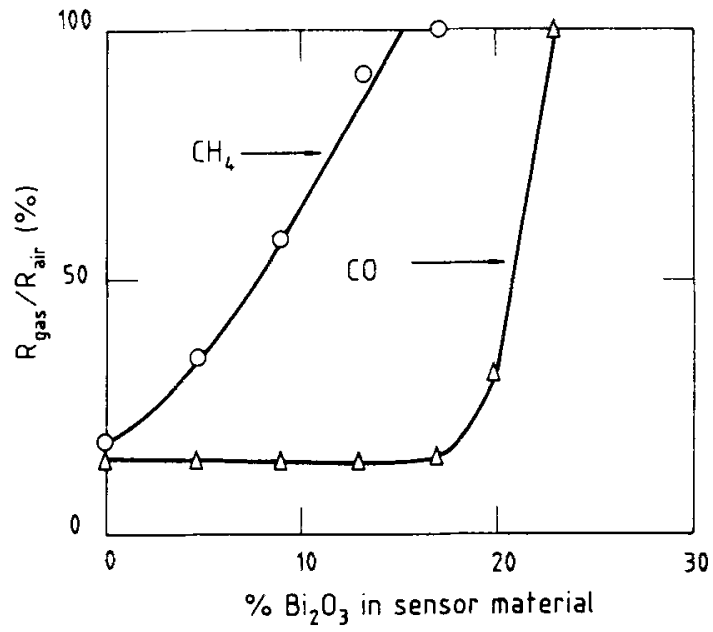

Figure 6. Variation of sensor sensitivity with increasing bismuth oxide content in atmospheres containing $1 \% \mathrm{CO}$ and $\mathrm{CH}_{4}$

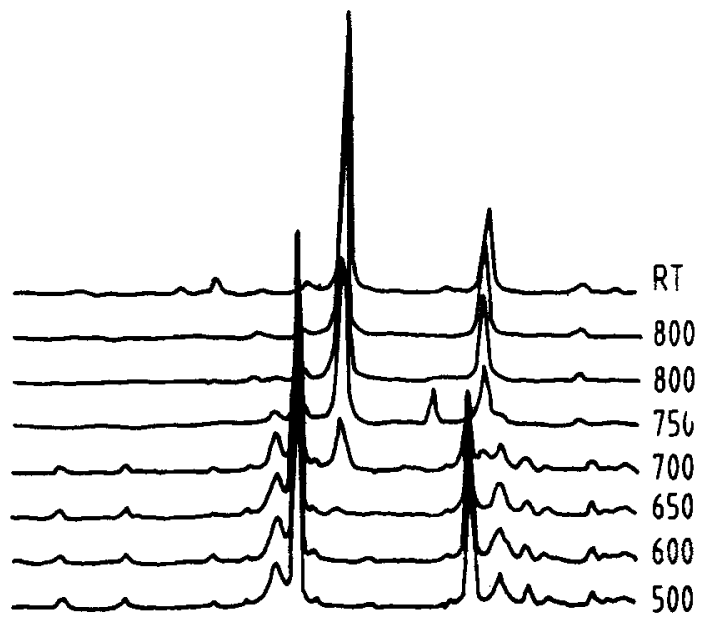

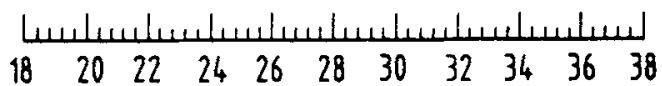

\section{2-Theta $I^{\circ}$}

Figure 7. X-ray diffraction patterns from a 2:1 mixture of tin dioxide and bismuth oxide with increasing temperature and after cooling to room temperature.

tin and bismuth oxides with increasing temperature. At temperatures below $600^{\circ} \mathrm{C}$ the peaks characteristic of these oxides are observed. At temperatures above this a peak is observed at an angle of $2 \theta=28 \cdot 6^{\circ}$. This corresponds to the production of bismuth stannate $\left(\mathrm{Bi}_{2} \mathrm{Sn}_{2} \mathrm{O}_{7}\right)$ (Roth 1956). At $750^{\circ} \mathrm{C}$ a peak is observed at an angle of 


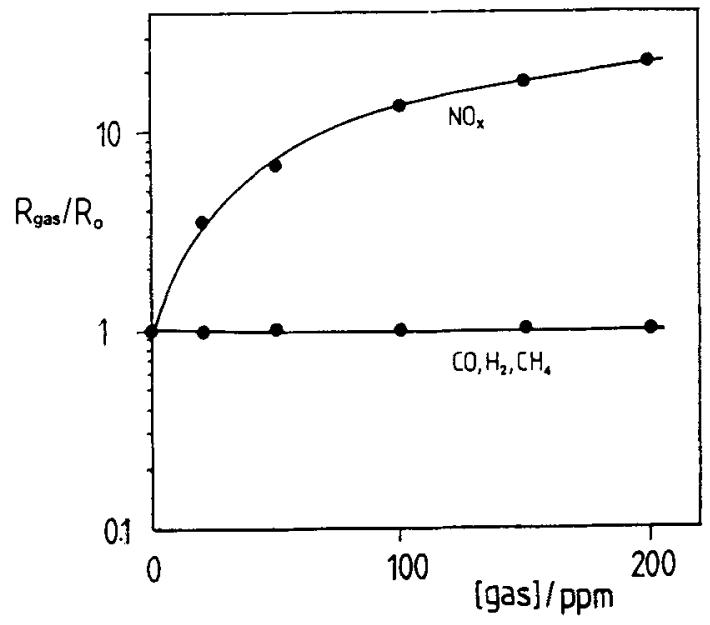

Figure 8. The response of an in-house $\mathrm{NO}_{x}$ sensor with increasing concentrations of $\mathrm{NO}_{2}$, $\mathrm{CO}, \mathrm{CH}_{4}$ and $\mathrm{H}_{2}$.

$2 \theta=31.4^{\circ}$ and this represents the phase transition of $\alpha \mathrm{Bi}_{2} \mathrm{O}_{3}$ to $\delta \mathrm{Bi}_{2} \mathrm{O}_{3}$ which is known to occur at a temperature of $\sim 710^{\circ} \mathrm{C}$. This peak has disappeared at higher temperatures. It can therefore be concluded that in the actual sensor, after sintering, all of the bismuth oxide has reacted and the sensor is composed of bismuth stannate and excess tin dioxide. The reasons why this material should confer the observed selectivity is unclear but it is known that bismuth stannate has a pyrochlore structure. In fact figure 7 also shows that at elevated temperatures the structure is that of a cubic pyrochlore which becomes a distorted pyrochlore on cooling to room temperature. This crystal form has a very open structure and it has been proposed that it may act as a molecular sieve on the surface of the tin dioxide particles. It should however be noted that evidence for this proposal is inconclusive and attempts to produce similar sensors incorporating other pyrochlore stannates have proved unsuccessful (Coles et al 1994).

The most recent sensor to be produced at Swansea selectively detects nitrogen dioxide in the presence of many other fuel and combustion gases (Coles and Williams 1993). This device is produced by depositing a layer of tin directly onto a substrate by radio frequency magnetron sputtering. This layer is then oxidized in air at elevated temperatures by simply powering the substrate heater. Figure 8 shows the response of this device in atmospheres containing $\mathrm{NO}_{2}, \mathrm{CO}, \mathrm{CH}_{4}$ and $\mathrm{H}_{2}$. Clearly this sensor shows a remarkable degree of selectivity for a material that is normally considered to respond to all of these gases. It is hoped that this device may prove useful in monitoring this environmentally sensitive gas in automotive applications such as exhaust monitoring from cars fitted with catalytic converters.

\section{Conclusions}

There can be no doubt that the world market for sensors of all types will continue to grow. This includes gas sensors that are required to monitor a wide variety of potentially explosive, toxic and environmentally sensitive gases. Although there are 
many technologies available for exploitation in this context, and which possess many commercially desirable characteristics, it is clear that semiconductor sensors will continue to hold many advantages over competing devices. At Swansea the problem of selectivity has been the main focus of work for over a decade and four sensors with marked selectivity have been developed. However, there are still many characteristics of these sensors that can be improved, major areas of concern being medium/ long-term drift, the ability to produce them reproducibly, and the effects of ambient conditions particularly humidity. These are all problems that are being studied by research groups around the world including our own. Although semiconductor devices have been available commercially for over 20 years there is little doubt that they will continue to form the basis of numerous publications and an integral part of various international conferences for many years to come.

\section{References}

Clifford P K and Tuma D T 1982/3 Sensors and Actuators 3233

Coles G S V, Gallagher K J and Watson J 1985 Sensors and Actuators 7, 2,89

Coles G S V, Williams G and Smith B 1991 Sensors and Actuators 3,1,7

Coles G S V and Williams G 1993 Sensors and Actuators B15-16 349

Coles G S V. Bond S E and Williams G 1994 J. Mater. Chem. 423

Firth $J$ G, Jones A and Jones T A 1975 Ann. Occup. Hyg. 1863

Harmer Associates European research on advanced sensors Report for the Department of Trade and Industry, UK

Morrison S R 1987 Sensors and Actuators 11283

Roth R S 1956 J. Res. Natl. Bur. Stand. (US) 5617 